\title{
An engineering model on penetration of eroding rods into ceramic/polymer composite
}

\author{
R. Zaera, A. Arias and C. Navarro \\ Department of Continuum Mechanics and Structural Analysis, Carlos III University of Madrid, \\ Avda. de la Universidad 30, 28911 Leganés, Madrid, Spain
}

\begin{abstract}
A new material composed of a polymer matrix loaded with ceramic particles, at low cost and of higher ballistic efficiency than that of the metallic materials used for amouring applications, is proposed as an altemative to the ceramic tiles traditionally adopted for mixed panels. An engineering model was developed to simulate normal impacts of projectiles against this particulate composite backed by a metallic plate. The equation governing the erosion of projectile and ceramic is that proposed by Tate and Alekseevskii, modifised to consider a moving target. For the volume of particulate composite between the projectile and the metallic plate, an equation was derived to include a momentum balance. For the metallic plate, an energy balance equation was used. When the projectile has eliminated the ceramic/polymer composite and meets the plate, the model of Awerbuch \& Bodner is adopted, again modified to consider a moving target. In this way, the impact process is described by a set of non-linear differential equations, to be integrated in time. The model was validated with experimental results and with full numerical simulations and showed agreement with both of them.
\end{abstract}

\section{INTRODUCTION}

The combination of the lightness and hardness of ceramic materials with the ductility of metallic materials in mixed protections provides high ballistic efficiency against the impact of low and medium-caliber projectiles. Although the development of advanced structural ceramics has brought an improvement in the mechanical properties, their application in mixed protection is limited by their high cost. A material that covers the gap between metallic armours and ceramic armours could be of great interest in applications for which cost saving is imperative. In a recent paper [1], we presented a material composed of ceramic particles and a polymeric matrix. The ballistic efficiency of tile of this composite backed by a metallic plate, measured as the areal density of the panel that can arrest a given projectile at a given impact velocity, is intermediate between that of aluminum plates and monolithic ceramic/aluminum panels. In the same paper, we proposed an accumulative damage model for this material, implemented in a numerical code to simulate the impact of a projectile against a protection made of the ceramic/polymer composite.

Numerical simulation codes allow a final tuning in the armour design process but their high computational cost makes them prohibitive in the early stages of design. For this task, a low-cost simulation tool is required that would solve a problem of impact in the shortest possible time, and with the required precision. In this respect, engineering models simulate the impact process by straightforward equations and parameters that can be assigned by simple experiments. Since they are quick and easy to use, they allow the simulation of a large number of impact problems, covering a large range of projectile/protection configurations, to achieve an optimal design.

This work presents a simplified engineering model for the simulation of the impact of a metallic projectite on a target of the new material developed. The model was validated with experimental and numerical resuits. 


\section{DESCRIPTION OF THE ENGINEERING MODEL}

\subsection{Projectile and eroding model}

Penetration into the intact ceramic is assumed to be governed by the Tate and Alekseevski model $[2,3]$. Since the target is not stationary, the velocity of the rear face of the composite tile $w$ is introduced into the modified Bernouilly equation, leading to the set of equations

$$
\begin{aligned}
Y_{p}+\frac{1}{2} \rho_{p}(v-u)^{2} & =Y_{c}+\frac{1}{2} \rho_{c}(u-w)^{2} \\
\frac{\mathrm{d} L}{\mathrm{~d} t} & =-(v-u) \\
\frac{\mathrm{d} v}{\mathrm{~d} t} & =-\frac{Y_{p}}{L \cdot \rho_{p}}
\end{aligned}
$$

where $Y_{p}$ is the dynamic yield stress of the projectile material, $Y_{\mathcal{C}}$ is the compressive strength of the composite, $\rho_{p}$ and $\rho_{c}$ are the densities of projectile and composite, $L$ the actual length of the projectile, $v$ its velocity and $u$ the penetration speed (see Figure 1).

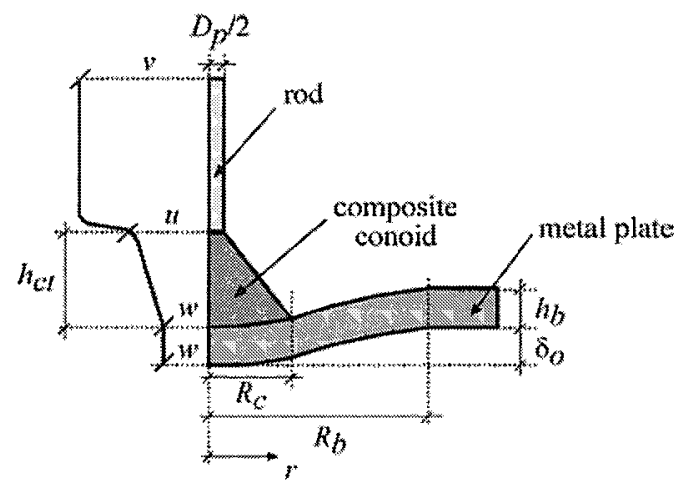

Figure 1. Velocity profile along impact axis.

\subsection{Composite material}

According to the hypothesis commonly assumed for monolithic ceramic armours [4,5], a conoid of the particulate composite material develops a few microseconds after impact, which pushes forward a circular area of the metallic plate with radius $R_{b}$ wider than the base of the composite conoid $R_{c}$ (Figure 1). If this conoid is a variable mass system, the linear momentum equation could be derived by assuming that the particles detached from the sides and from the erosion surface maintain the same velocity in the impact direction, so that the control volume in the equation would be the corresponding to the cone at the instant $t+\Delta t$ (Figure 2). Assuming a linear variation of velocity between $u$ and $w$ (Figure 2),

$$
\begin{aligned}
& \sigma_{i} \pi \frac{D_{p}^{2}}{4}-f_{b} \pi R_{c}^{2}=\pi \rho_{c} h_{c t}\left[\dot{u}\left(\frac{D_{p}^{2}}{16}+\frac{R_{c}^{2}}{12}+\frac{D_{p} R_{c}}{16}\right)+\dot{w}\left(\frac{D_{p}^{2}}{48}+\frac{R_{c}^{2}}{4}+\frac{D_{p} R_{c}}{12}\right)\right]+ \\
& +\pi \rho_{c} \dot{h}_{c t}\left[u\left(\frac{7 D_{p}^{2}}{48}+\frac{5 R_{c}^{2}}{12}\right)-w\left(\frac{D_{p}^{2}}{16}+\frac{13 R_{c}^{2}}{12}+\frac{D_{p} R_{c}}{12}\right)\right]+\pi \rho_{c} h_{c t} \dot{R}_{c}\left[u\left(\frac{D_{p}}{12}+\frac{R_{c}}{6}\right)+w\left(\frac{D_{p}}{6}+\frac{R_{c}}{12}\right)\right]
\end{aligned}
$$


where $D_{p}$ is the projectile diameter, $f_{b}$ the compressive stress at the conoid-metal plate interface, $h_{c t}$ the conoid thickness and $\sigma_{c}$ the compressive stress at the projectile-composite interface (Figure 2) which could be calculated according to the Tate model

$$
\sigma_{c}=\left\{\begin{array}{cc}
Y_{c} & \text { conoid erosion } \\
Y_{p}+\frac{1}{2} \rho_{p}(v-w)^{2} & \text { no conoid erosion }
\end{array}\right.
$$

The base radius of the conoid is derived from the expression

$$
R_{c}=D_{p} / 2+h_{c t} \tan (\alpha)
$$

$\alpha$ being the conoid semiangle (Figure 2). Measurements of the composite fragments recovered after impact gave the value of $\alpha$ around $55^{\circ}$.

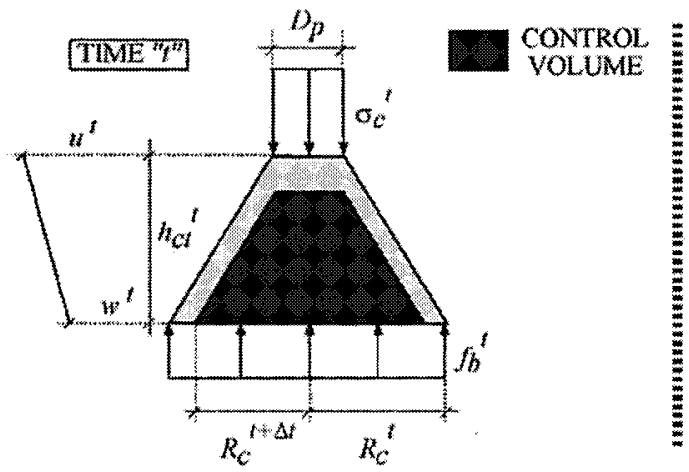

Figure 2. Approach to the linear momentum equation for the composite material conoid.

\subsection{Metallic plate}

We developed recently an analytical model to simulate the dynamic response of metallic circular plates subjected to impulsive loads [6]. It is based on the plate energy balance equation

$$
\dot{W}_{E}=\dot{E}_{K}+\dot{W}_{Y}
$$

in which $W_{E}, E_{K}$ and $W_{Y}$ are the external work, the kinetic energy and the plastic work. Each term was obtained by proposing for the plate a displacement field of the form

$$
\delta(r, t)=\delta_{o}(t) \phi(r)
$$

where $\delta_{O}$ is the central point displacement $\left(d \delta_{o} / d t=w\right)$ and $\phi$ a shape function of the radial coordinate (Figure 1). The power of external forces can be expressed as

$$
\dot{W}_{E}=2 \pi f_{b} \int_{0}^{R_{C}} \dot{\delta}_{o} \phi r d r
$$

and the time derivative of kinetic energy (taking translation and rotation terms into account) is

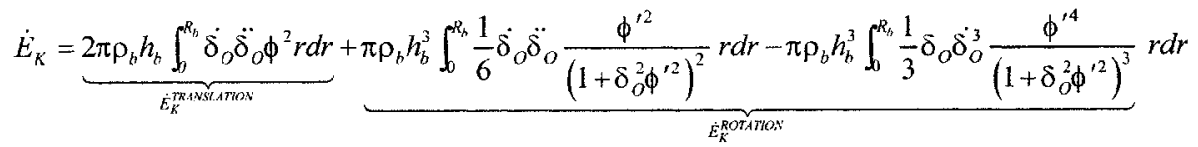


where $h_{b}$ is the metal plate thickness. Plastic power is obtained by adding in-plane stretching $\dot{W}_{Y}^{S}$ and curvature terms $\dot{W}_{Y}^{R}, \dot{W}_{Y}^{\theta}$

$$
\dot{W}_{Y}=\underbrace{2 \pi \int_{0}^{R_{h}} \delta_{O} \dot{\delta}_{O} N_{r} \frac{\phi^{\prime 2}}{\left(1+\delta_{o}^{2} \phi^{\prime 2}\right)^{1 / 2}} r d r}_{\psi_{Y}^{S}}+\underbrace{2 \pi \int_{0}^{R_{n}} M_{r}\left(\chi_{r} \dot{d} s+\dot{\chi}_{r} d s\right)}_{W_{r}^{R}} r+\underbrace{2 \pi \int_{b}^{R_{b}} M_{\theta} \dot{\chi}_{\theta} r d r}_{H_{r}^{\theta}}
$$

in which $N_{r}, M_{r}$ and $M_{\theta}$ are the membrane force, the radial bending moment and the circumferential bending moment per unit length. Curvatures are determined from the expressions

$$
\begin{gathered}
\chi_{r}=-\frac{\delta_{o} \phi^{\prime \prime}}{\left(1+\delta_{o}^{2} \phi^{\prime 2}\right)^{3 / 2}} \\
\chi_{\theta}=-\frac{\delta_{o} \phi^{\prime}}{r\left(1+\delta_{o}^{2} \phi^{\prime 2}\right)^{1 / 2}}
\end{gathered}
$$

and the differential radial length at mid-plane is

$$
d s=\left(1+\delta_{o}^{2} \phi^{\prime 2}\right)^{1 / 2} d r
$$

Internal forces are obtained by applying the normality rule of plasticity to the yield criterion

$$
\left(\frac{N_{r}}{N_{Y}}\right)^{2}+\left(\frac{M_{r}}{M_{Y}}\right)^{2}+\left(\frac{M_{\theta}}{M_{y}}\right)^{2}-\frac{M_{r} M_{\theta}}{M_{y}^{2}}=1
$$

where $N_{Y}$ and $M_{Y}$ are the fully plastic membrane force and the bending moment,

$$
\begin{gathered}
N_{Y}=\sigma_{Y b} h_{b} \\
M_{Y}=\sigma_{Y b} h_{b}^{2} / 4
\end{gathered}
$$

and $\sigma_{Y b}$ the metal plate yield stress. On penetration of the projectile into the metal plate, the lower limit of the integrals should be the projectile radius $D_{p} / 2$, thus neglecting the effect of the part of the metal plate ahead of the projectile.

\subsection{Penetration in the metallic backing plate}

When the projectile has eliminated the composite cone, it meets the plate and penetrates it. For this stage, we used the model of Awerbuch and Bodner [7] to compute deceleration of the projectile. Because this model was derived for stationary targets, their equations were adjusted to take in the movement of the plate axis. Deceleration of the projectile is derived as follows

$$
\frac{d v}{d t}=-\frac{F_{p}+\frac{d m_{p h}}{d t}(v-w)}{\rho_{p} L A_{p}+m_{p h}}
$$

where $F_{p}$ is the force resisting penetration, $A_{p}$ the cross section area of the projectile $\left(A_{p}=\pi D_{p}{ }^{2} / 4\right)$ and $m_{p b}$ the plate mass being continuously added to the projectile tip

$$
m_{p b}=\rho_{b} A_{p} z_{p}
$$

$z_{p}$ being the penetration into the plate

$$
\frac{d z_{p}}{d t}=(v-w)
$$


For the first stage of the model, $F_{p}$ is given by

$$
F_{p}=\frac{1}{2} \rho_{b} A_{p}(v-w)^{2}+\sigma_{\gamma_{b}} A_{p}
$$

and for the second stage

$$
F_{p}=\frac{1}{4} \rho_{h} A_{p}(v-w)^{2}+\sigma_{Y b} A_{p}\left[1-\left(\frac{z_{p}-h_{b}+b}{b}\right)^{2}\right]+\pi D_{p}\left(z_{p}-h_{b}+b\right)\left[\tau_{o}+\frac{\mu}{e}(v-w)^{2}\right]
$$

where $b$ is a fraction of the plate thickness $\left(b / h_{b}=0.715\right.$ for aluminium plate), $\tau_{o}$ the ultimate shear stress of the metal plate, and $\mu$ and $e$ constants of the Awerbuch and Bodner model. For the third stage

$$
F_{p}=\pi D_{p}\left(h_{b}-z_{p}\right)\left[\tau_{o}+\frac{\mu}{e}(v-w)^{2}\right]
$$

During penetration of the projectile into the metal plate, $f_{b}$ in equation (9) takes the value $f_{b}=F_{p} / A_{p}$. If the energy of the projectile is high, it will perforate the panel; if not, it will be arrested by the armour.

\section{MODEL VALIDATION}

To validate the model, experimental tests were made by firing cylindrical projectiles (AISI 52100 chrome alloy steel, $D_{p}=5.3 \mathrm{~mm}, L_{0}=7 \mathrm{~mm}, Y_{p}=1.2 \mathrm{GPa}$ following the recommendations of Tate [2], $\left.\rho_{p}=7850 \mathrm{~kg} / \mathrm{m}^{3}\right)$ against composite tiles $\left(h_{c}=4.8 \mathrm{~mm}, \rho_{c}=2463 \mathrm{~kg} / \mathrm{m}^{3}\right)$ backed by a metallic plate (2017-T6 aluminium alloy, $h_{b}=1.5 \mathrm{~mm}, \sigma_{Y b}=230 \mathrm{MPa}, \rho_{b}=2790 \mathrm{~kg} / \mathrm{m}^{3}, \tau_{c}=126 \mathrm{MPa}, \mu=0.9$ $10^{-3} \mathrm{MPa} \cdot \mathrm{s}, \mathrm{e}=0.53 \mathrm{~mm}$ ). Grains of alumina (ALCOA T-60) of $99.4 \%$ purity and a vinylester resin (Plastiform Epovia RF-1001) were mixed to manufacture the composite tiles, using two different proportions of grain size (Table 1). The values of $Y_{C}$ were determined by calibrating the Tate-Alekseevskii model with results of Depth of Penetration Tests [8] performed for both composites.

Table 1. Proportions of grain size in manufactured tiles $(\tilde{\delta}=$ mean grain size).

\begin{tabular}{|l|c|c|c|c|c|}
\hline & {$[0-1]$} & {$[1-3] \mathrm{mm}$} & {$[3-6] \mathrm{mm}$} & $\delta$ (mm) & $Y_{c}(\mathrm{MPa})$ \\
\hline Large Grain composite & $20 \%$ & $30 \%$ & $50 \%$ & 2.0 & 1290 \\
\hline Small Grain composite & $50 \%$ & $30 \%$ & $20 \%$ & 1.3 & 1025 \\
\hline
\end{tabular}

Impact velocities ranged from 390 to $570 \mathrm{~m} / \mathrm{s}$, the ballistic limit lying around $500 \mathrm{~m} / \mathrm{s}$. Impact simulations below the ballistic limit were validated with the experimental results of plate axis permanent displacement, whereas simulations above the ballistic limit were validated with the residual velocity determined by full numerical simulation (using the damage model presented in [1] for the ceramic/polymer composite). Figure 3 illustrates the validation of the two types of composite. The ballistic limit indicated by the model is fairly accurate, and the numerical elements chosen for the validation are close enough to the reference values adopted in the predictions. 

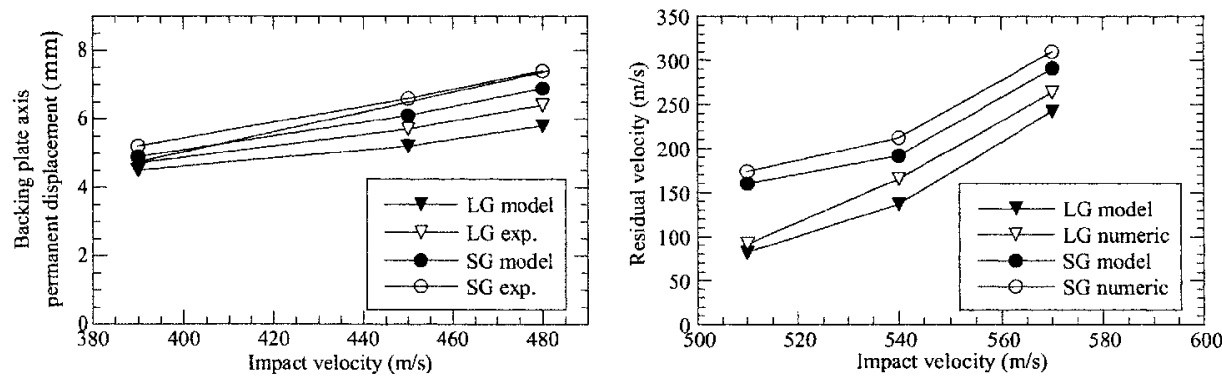

Figure 3. Results of the engineering model compared with experimental results (left) and with numerical results (right) of Small Grain composite (SG) and Large Grain composite (LG).

\section{CONCLUSIONS}

A simplified model was made to simulate the high velocity impact of a cylindrical projectile on an armour panel made of a tile of a particulate-loaded ceramic tile and a metallic plate. The model, based on simple erosion, momentum and energy balances, leads a set of non-linear differential equations to be solved numerically. Time histories of the projectile mass and velocity and of the backing plate displacement were obtained in a few seconds, allowing the simulation of a large number of impact problems in a short time. The ballistic limits given by the model are close to the experimental ones.

\section{Acknowledgements}

The authors are indebted to the Comunidad Autónoma de Madrid (Project 07N/0004/1998) for the financial support of this work.

\section{References}

[1] Arias A., Zaera R., López-Puente J., Navarro C., "Numerical modeling of the impact behavior of new particulate-loaded composite materials", Compos. Struct, (in press).

[2] Tate A., "A theory for the deceleration of long rods after impact", J. Mech. Phys. Solids 15 (1967) pp. 387-399.

[3] Alekseevskii V. P., "Penetration of a rod into a target at high velocity", Combustion, explosion and shock waves (Faraday Press, New York, 1966).

[4] Shockey D. A., Marchand A. H., Skaggs S. R., Cort G. E., Burkett M. W., Parker R., "Failure phenomenology of confined ceramic targets and impacting rods", Int. J. Impact Engng. 9:3 (1990) pp. 263-275.

[5] Riou P., Beylat L., Cottenot C., Derep J. L., "Impact damage on silicon carbide: first results", J. Physique IV 4 (1994) pp. 281-287.

[6] Zaera R., Arias A., Navarro C., "Analytical modelling of metallic circular plates subjected to impulsive loads", Int. J. Solids Struct. 39 (2002) pp. 659-672.

[7] Awerbuch J., Bodner S. R., "Analysis of the mechanics of perforation of projectiles in metallic plates", Int. J. Solids Struct. 10 (1974) pp. 671-684.

[8] Hohler V., Stilp A. J., Weber K., "Hypervelocity penetration of tungsten sinte-alloy rods into alumina", Int. J. Impact Engng. 17 (1995) pp 409-418. 